\title{
Effects of Tanshinone IIA on the modulation of miR-33a and the SREBP-2/Pcsk9 signaling pathway in hyperlipidemic rats
}

\author{
LIANQUN JIA ${ }^{1}$, NAN SONG ${ }^{1}$, GUANLIN YANG ${ }^{1}$, YIXIN MA ${ }^{2}$, XUETAO LI $^{1}$, REN LU $^{1}$, HUIMIN CAO $^{3}$,

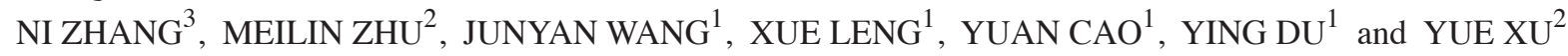 \\ ${ }^{1}$ Key Laboratory of Ministry of Education for Traditional Chinese Medicine Viscera-State Theory and Applications; \\ ${ }^{2}$ Graduate School; ${ }^{3}$ The First Clinical College, Liaoning University of Traditional Chinese Medicine, \\ Shenyang, Liaoning 110847, P.R. China
}

Received April 2, 2015; Accepted February 26, 2016

DOI: $10.3892 / \mathrm{mmr} .2016 .5133$

\begin{abstract}
Tanshinone IIA is the active compound isolated from Salvia miltiorrhiza bunge, which is a traditional Chinese medicine known as Danshen. The aim of the present study was to assess the effect of Tanshinone IIA on the regulation of lipid metabolism in the livers of hyperlipidemic rats and the underlying molecular events. An in vivo model of hyperlipidemia was established in rats, with the animals receiving a daily dose of Tanshinone IIA. The serum lipid profiles were analyzed using an automatic biochemical analyzer, and the histopathological alterations and lipid deposition in liver tissue were assessed using hematoxylin and eosin staining, and oil red $\mathrm{O}$ staining, respectively. The mRNA expression levels of microRNA (miR)-33a, ATP-binding cassette transporter (ABC)A1, ABCG1, sterol regulatory element-binding protein 2 (SREBP-2), proprotein convertase subtilisin/kexin type 9 (Pcsk9) and low-density lipoprotein receptor (LDL-R) in liver tissues were measured using reverse transcription-quantitative polymerase chain reaction, and the protein expression levels of ABCA1, ABCG1, SREBP-2, Pcsk9, and LDL-R were analyzed using western blotting. Tanshinone IIA reduced lipid deposition and improved histopathology in the rat liver tissue, however, did not alter the lipid profile in rat serum. In addition, Tanshinone IIA treatment suppressed the expression of miR-33a, whereas the protein expression levels of ABCA1, SREBP-2, Pcsk9 in addition to LDL-R mRNA and protein were upregulated. In conclusion, the present study indicated that Tanshinone IIA attenuated lipid deposition in the livers of
\end{abstract}

Correspondence to: Professor Guanlin Yang, Key Laboratory of Ministry of Education for Traditional Chinese Medicine Viscera-State Theory and Applications, Liaoning University of Traditional Chinese Medicine, 79 Chongshan Eastern Road, Huanggu, Shenyang, Liaoning 110847, P.R. China

E-mail: yang_guanlin@163.com

Key words: hyperlipidemia, Tanshinone IIA, miR-33a, SREBP-2 Pcsk9 hyperlipidemic rats and modulated the expression of miR-33a and SREBP-2/Pcsk9 signaling pathway proteins.

\section{Introduction}

Hyperlipidemia refers to abnormally elevated levels of lipids or lipoproteins in the bloodstream, and is a significant risk factor associated with numerous diseases, including ventricular remodeling, cardiac hypertrophy and cardiac dysfunction (1). A previous study demonstrated that hyperlipidemia has a direct association with an increase in the number and size of atheromatous plaques and thus has been considered as a contributor to atherosclerosis (2). Patients with hyperlipidemia usually have higher levels of total cholesterol (TC), triglycerides (TG), and low-density lipoprotein cholesterol (LDL-C), however, relatively lower levels of high-density lipoprotein cholesterol (HDL-C) compared with healthy controls (3). High plasma TC, TG or LDL-C levels or low plasma HDL-C levels may result in damage to vascular endothelial cells and initiates atherosclerosis (4). By contrast, HDL-C has been generally accepted to serve a protective role in the suppression of the formation of atherosclerosis (5). Thus, clinical control of hyperlipidemia may prevent atherosclerosis and cardiovascular diseases.

Tanshinone IIA is a pharmacologically active compound isolated from Salvia miltiorrhiza bunge, and is known as Danshen in traditional Chinese medicine. Tanshinone IIA has been used historically in traditional Chinese medicine to prevent and treat cardiovascular diseases in Asian countries, due to its putative cardioprotective and anti-atherosclerotic effects. Numerous experimental and clinical studies have indicated that Tanshinone IIA possesses a variety of biological activities, including, but not limited to, vasodilatory, anti-atherosclerosis, anti-oxidation, anti-inflammatory, anti-hyperlipidemic and anti-adipogenic effects (6-9). However, the underlying mechanisms of Tanshinone IIA activity remain to be determined. In a previous study, Tanshinone IIA treatment increased HDL-C level and reduced LDL-C levels in C57BL/6J mice fed with a high fat-diet (9). Additionally, Tanshinone IIA has been shown to reduce the formation of atherosclerotic plaques without a significant effect on alterations in the lipid profile $(6,10)$. A previous study indicated that 
Tanshinone IIA served an anti-atherosclerotic effect via the inhibition of Toll-like receptor 4 and tumor necrosis factor- $\alpha$ expression in EA.hy926 cells following stimulation with lipopolysaccharides (11). Additionally, protective effects of Tanshinone IIA have been indicated on apoptosis induced by $\mathrm{H}_{2} \mathrm{O}_{2}$ in EA.hy926 cells (12).

Increasingly, evidence indicates that sterol regulatory element-binding protein 2 (SREBP-2) and LDL receptor (LDL-R) are transcription factors involved in the regulation of cholesterol metabolism (13-15). Furthermore, there is a binding site for SREBP localized in the promoter region of proprotein convertase subtilisin/kexin type 9 (Pcsk9) (16) and thus, SREBP regulates expression of Pcsk9 at the transcriptional level. Reductions in hepatic cholesterol levels are able to activate the SREBP signaling pathway to upregulate the expression of Pcsk9 and LDL-R simultaneously $(15,17)$, and in turn, increase the levels of LDL particles by reducing LDL-R (18). Conversely, inhibition of SREBP expression in the liver has been shown to reduce the levels of Pcsk9 and LDL-R (19). In addition, a previous study indicated that microRNA (miR)-33, an intronic miRNA residing in the SREBP-2 gene, serves a crucial role in the regulation of HDL metabolism, cholesterol efflux and fatty acid $\beta$-oxidation through the modulation of the expression of the ATP-binding cassette transporter A1 (ABCA1) and G1 (ABCG1) (20-23). However, in mice, but not in humans, miR-33 overexpression has been observed to reduce cholesterol efflux to nascent HDL (24). Thus, inhibition of endogenous miR-33 levels may possess therapeutic function to upregulate HDL levels by repressing ABCA1 and ABCG1 expression $(25,26)$. Thus, in the present study, experiments were conducted to elucidate the potential mechanisms of Tanshinone IIA activity in vivo on lipid metabolism and the underlying molecular events.

\section{Materials and methods}

Animals and rat hyperlipidemia model. The study was approved by the ethics committee of Liaoning University of Traditional Chinese Medicine experimental animal ethics committee (Shenyang, China). A total of 90 male Sprague-Dawley rats (6 weeks old, 200 \pm 10 g) were obtained from Vital River Laboratories Co., Ltd. (Beijing, China) and housed in a climate-controlled environment $\left(22 \pm 1^{\circ} \mathrm{C}\right.$, humidity at $\left.50 \pm 5 \%\right)$ with a $12 / 12 \mathrm{~h}$ light/dark cycle and ad libitum access to water. The rats were randomly divided into control, hyperlipidemia (HLP) and Tanshinone IIA treatment (TAN) groups, with 30 rats in each group. The control rats were fed with a regular balanced diet, while the HLP and TAN groups were fed with a high-fat diet (6\% sucrose, $1 \%$ sodium glutamate, $5 \%$ yolk powder, $8 \%$ peanut oil, $1.5 \%$ cholesterol, $0.4 \%$ methylthiouracil, $0.2 \%$ cholate and $73.3 \%$ regular balanced diet) for 3 months. Following this, the TAN group received $1.2 \mathrm{ml}$ of sodium Tanshinone IIA sulfonate (Shanghai No. 1 Biochemical \& Pharmaceutical Co., Ltd., Shanghai, China) at a dose of $10 \mathrm{mg} / \mathrm{kg}$ by daily intraperitoneal injection for an additional 3 months, whereas the control and HLP groups received the same amount of phosphate-buffered saline (PBS) for the same period of time.

Collection of sera and lipid profiling. Following the treatment process, the rats $(n=6)$ were anesthetized with $10 \%$ chloral hydrate and aortic blood was collected for $2 \mathrm{~h}$ at room temperature. The blood samples were then centrifuged at $4^{\circ} \mathrm{C}$, $300 \mathrm{x}$ g for $20 \mathrm{~min}$, and the serum was collected and stored at $-80^{\circ} \mathrm{C}$ until use. For the lipid profiling, the serum samples were analyzed using an automatic TBA-120FR biochemical analyzer (Toshiba Corporation, Tokyo, Japan). Levels of TG, TC, HDL-C and LDL-C in the serum were measured using the corresponding kits (all from Sichuan Maker Biotechnology Co., Ltd., Sichuan, China).

Hematoxylin and eosin $(H \& E)$ staining of liver tissues. Liver tissues were collected $(n=3)$, fixed in $4 \%$ formaldehyde for $24 \mathrm{~h}$ at $4^{\circ} \mathrm{C}$, dehydrated through an ethanol series (70-100\%), cleared in xylene and embedded in paraffin. The tissue was cut into $5 \mu \mathrm{m}$ thick sections and dewaxed in xylene, rehydrated through a 70-100\% ethanol series, and then stained with hematoxylin, differentiated in $70 \%$ acid ethanol and further stained with eosin. Subsequently, the tissue sections were dehydrated through 70-100\% ethanol, cleared in xylene, and mounted using Permount Mounting Medium (BioWorld Technology, Inc., Atlanta, Georgia, USA). The tissue sections were photographed using a BX51-WIF light microscope linked to a digital charge-coupled device (CCD) camera (Olympus Corporation, Tokyo, Japan).

Oil Red O staining of liver tissues. For Oil Red O staining, frozen sections were prepared $(6 \mu \mathrm{m})$ from liver tissue samples $(n=3)$ and fixed in $50 \%$ ethanol. Subsequently, sections were stained with Oil Red O (Beijing Noble Rider Technology Co., Ltd., Beijing, China) for $8 \mathrm{~min}$ and differentiated with $50 \%$ ethanol, rinsed with tap water, and counterstained with hematoxylin. Following a final rinse in tap water, the sections were mounted with glycerin jelly. The sections were photographed using the light microscope linked to a digital CCD camera (Olympus Corporation).

Immunohistochemistry. The expression of SREBP-2, Pcsk9, and LDL-R proteins was assessed in paraffin-embedded liver tissues. In brief, tissue sections were dewaxed in xylene, rehydrated through a 70-100\% ethanol series, and then incubated in $0.3 \% \mathrm{H}_{2} \mathrm{O}_{2}$ in PBS to block endogenous peroxidase activity and $10 \%$ skim milk in PBS to block non-specific protein binding of the secondary antibodies. Subsequently, the sections were incubated with primary polyclonal rabbit anti-rat SREBP-2 (cat no. 980594w), LDL-R (cat no. YSLS15w), PCSK9 (cat no. bs-6060R) antibodies (all from BIOSS, Beijing, China). at $4^{\circ} \mathrm{C}$ overnight, then washed with PBS three times and incubated with a horseradish peroxidase (HRP)-conjugated goat anti-rabbit secondary antibody $(1: 1,000 ; 36 J 00180$; Beijing Dingguo Changsheng Biotechnology Co., Ltd., Beijing, China) at room temperature for $1 \mathrm{~h}$. The peroxidase activity was detected using 3, 3'-diaminobenzidine tetrahydrochloride solution. Following washing and counterstaining with hematoxylin, the sections were mounted using AquaMount [Hyde Entrepreneurship (Beijing) Biological Technology Co., Ltd., Beijing, China] and photographed and scored using the light microscope linked to a digital CCD camera (Olympus Corporation). The area of positive expression of each section was observed at the low magnification (x10), then 4 randomly selected fields were 
A
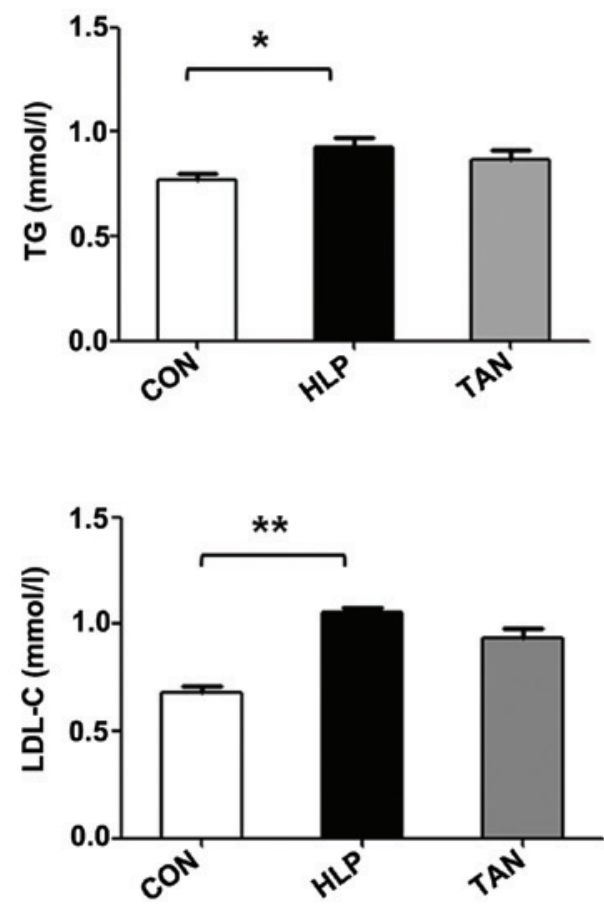
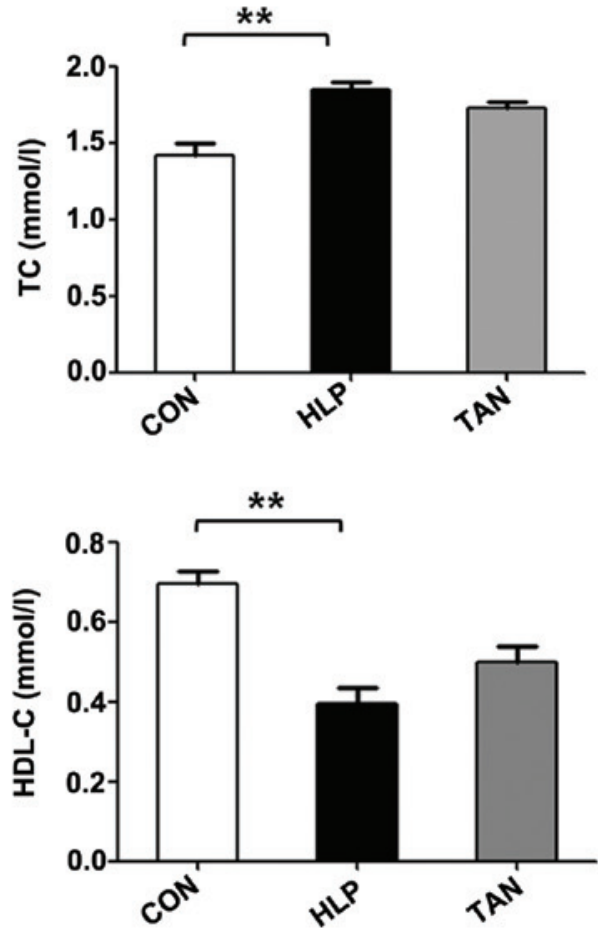

B
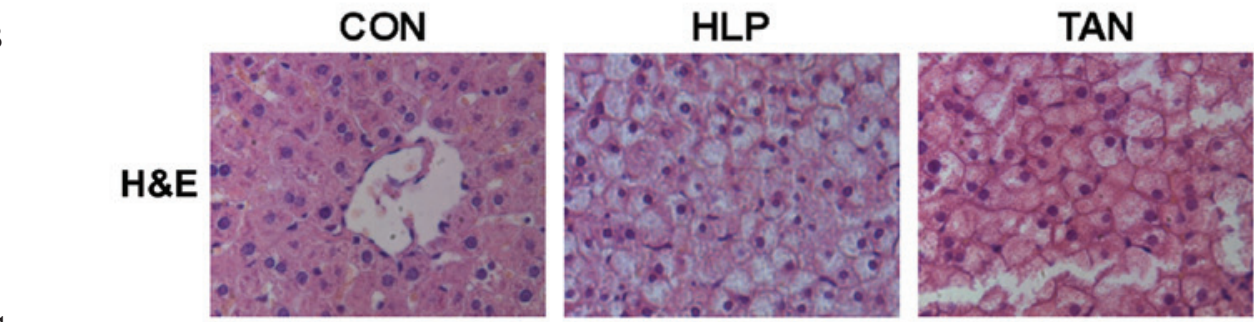

C
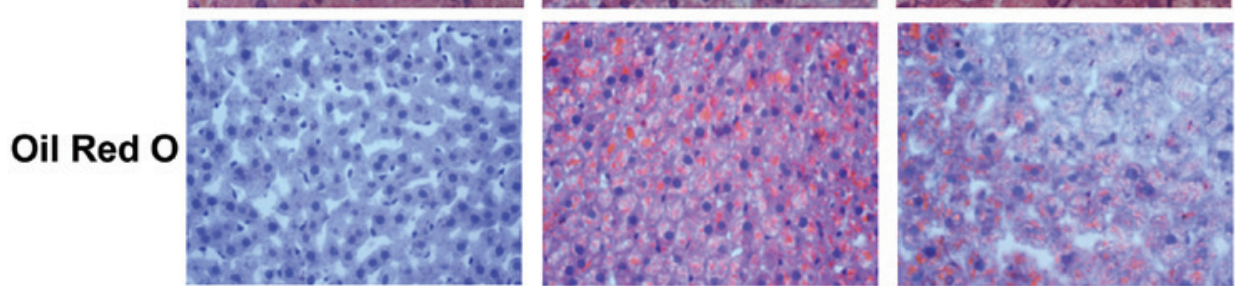

Figure 1. Tanshinone IIA alterations in the lipid levels in sera and lipid deposition in the liver. (A) TG, TC, LDL-C and HDL-C serum lipid levels. (B) H\&E staining indicating the alterations in the liver cells, such as lipid droplets. (C) Oil red O staining showing the lipid droplets in the liver tissue. Data are presented as the mean \pm standard deviation. ${ }^{*} \mathrm{P}<0.05,{ }^{* * *} \mathrm{P}<0.01$ vs. $\mathrm{CON}$ group. TG, triglycerides; TC, total cholesterol; LDL-C, low-density lipoprotein-cholesterol; HDL-C, high-density lipoprotein-cholesterol; H\&E, hematoxylin and eosin; CON, control; HLP, high lipid diet-fed rats; TAN, Tanshinone IIA treatment of high lipid diet-fed rats.

captured at high magnification (x40). Exposure conditions were consistent. Images were semi-quantitatively analysed using Image-Pro Plus software (Media Cybernetics, Inc., Rockville, MD, USA). The the integrated optical density of each image was measured and an average from 4 images was calculated for each section.

RNA isolation and reverse transcription-quantitative polymerase chain reaction (RT- $q P C R)$. Total RNA was isolated from liver tissue ( $\mathrm{n}=3$ ) using a TRIzol reagent (Takara Biotechnology Co., Ltd., Dalian, China) and reverse transcribed into cDNA using the miScript II RT kit (Qiagen GmbH, Hilden, Germany), according to the manufacturer's instructions. To assess the levels of miR-33, total RNA was isolated using a miRNeasyMini kit (Qiagen $\mathrm{GmbH}$ ), amplified using specific primers to miR-33 and normalized to small U6 RNA (Qiagen $\mathrm{GmbH}$ ). The miR-33 transcripts were amplified as follows: Initial denaturation at $94^{\circ} \mathrm{C}$ for $10 \mathrm{~min} ; 35$ cycles of denaturation at $94^{\circ} \mathrm{C}$ for $1 \mathrm{~min}$, annealing at $55^{\circ} \mathrm{C}$ for $15 \mathrm{sec}$ and extension at $70^{\circ} \mathrm{C}$ for $15 \mathrm{sec}$; and a final extension step at $70^{\circ} \mathrm{C}$ for $5 \mathrm{~min}$. To measure the levels of gene transcripts, the following rat primers were used: LDL-R, foward 5'-GGGTTCTTGTCCATCTTCC-3' and reverse 5'-ACT GGGTTGTCAAAGTTTATG-3'; SREBP-2, forward 5'-GTG GGCTGAGAAGAAAGATG-3' and reverse 5'-CCAGAGGCA GAAAGGAGAC-3'; and glyceraldehyde 3-phosphate dehydrogenase (GAPDH), forward 5'-TGTGTCCGTCGTGGATCT 
$\mathbf{A}$
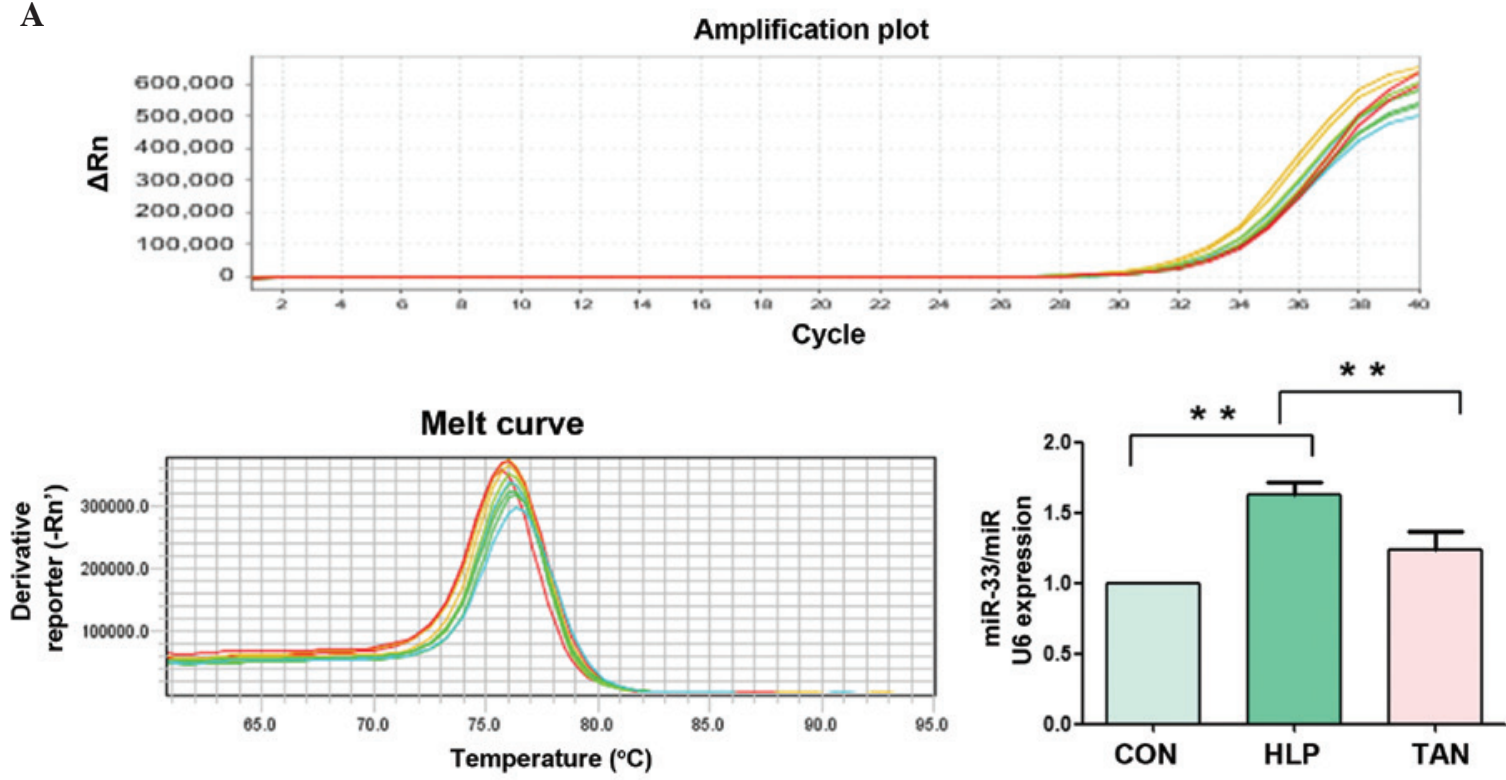

B
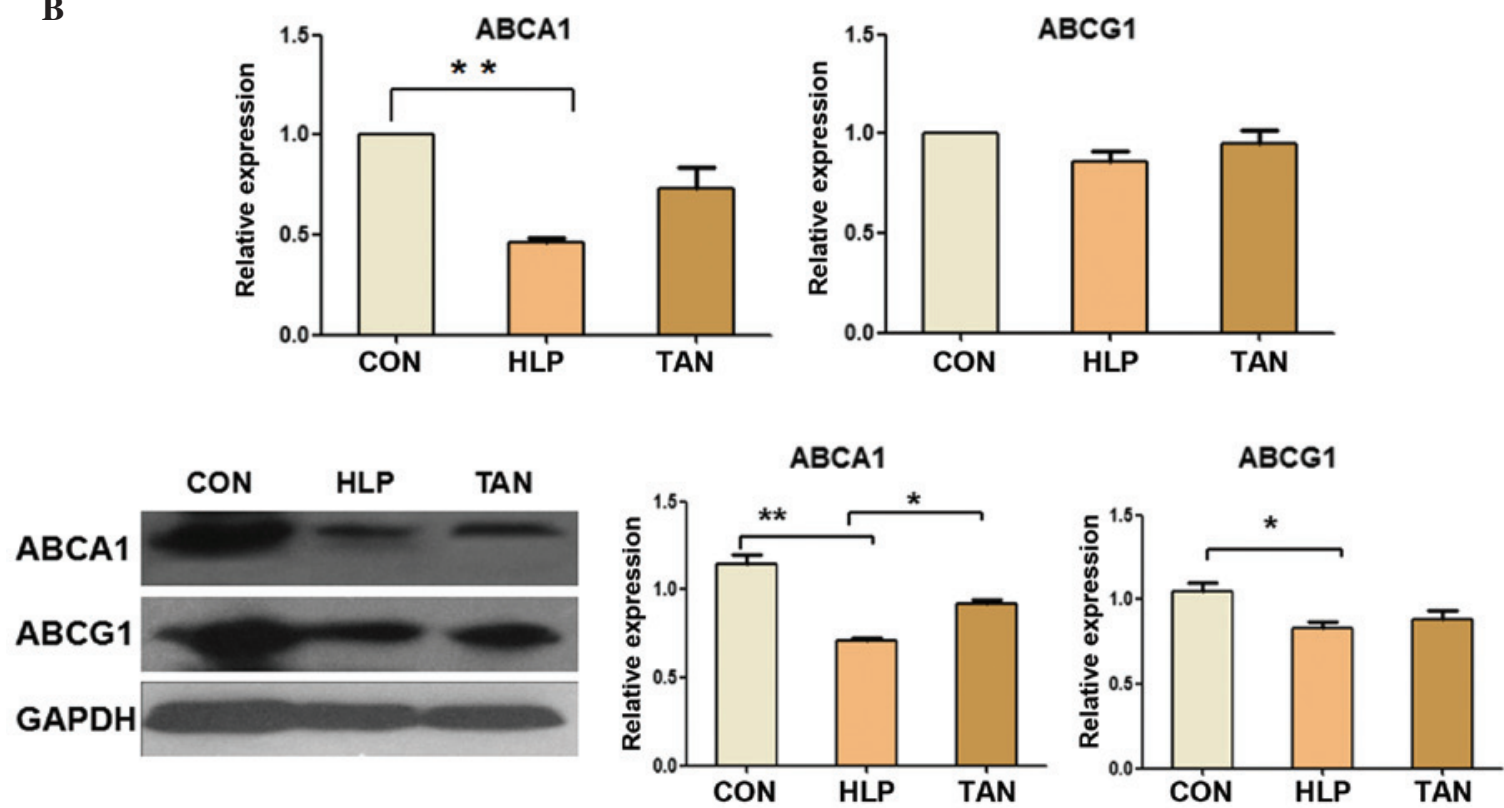

Figure 2. Tanshinone IIA regulation of miR-33a, ABCA1 and ABCG1 expression in the liver. (A) RT-qPCR measurement of miR-33a expression in rat liver tissue. (B) RT-qPCR and western blotting analyses of ABCA1 and ABCG1 expression in liver tissue. Data are presented as the mean \pm standard deviation. "P<0.05, ${ }^{* *} \mathrm{P}<0.01$. miR, microRNA; ABCA1, ATP-binding cassette transporter A1; ABCG1, ATP-binding cassette transporter G1; RT-qPCR, reverse trancription-polymerase chain reaction; CON, control; HLP, high lipid diet-fed rats; TAN, Tanshinone IIA treatment of high lipid diet-fed rats; GAPDH, glyceraldehyde 3-phosphate dehydrogenase.

GA-3' and reverse 5'-TTGCTGTTGAAGTCGCAGGAG-3'. All primers were designed using Premier 5.0 software (PREMIER Biosoft, Palo Alto, CA, USA) and custom-synthesized by Takara Biotechnology Co., Ltd. RT-qPCR was performed in triplicate using a SYBR Green Master Mix (Takara Biotechnology Co., Ltd.) in an ABI 7500 PCR Sequence Detector (Applied Biosystems; Thermo Fisher Scientific, Inc., Waltham, MA, USA). The transcripts were amplified by 40 cycles of $95^{\circ}$ for $2 \mathrm{~min}, 95^{\circ} \mathrm{C}$ for $30 \mathrm{sec}$ and $60^{\circ} \mathrm{C}$ for $30 \mathrm{sec}$. The gene transcripts were normalized to GAPDH. The RNA levels measured by RT-qPCR were quantatified used the $2^{-\Delta \Delta C q}$ method.

Protein extraction and western blotting. Total cellular protein was extracted from rat liver tissues $(\mathrm{n}=3)$ using radio- immunoprecipitation assay lysis buffer (Beyotime Institute of Biotechnology, Shanghai, China) and the protein concentration was measured using Bicinchoninic Acid Protein Assay kit (Beijing Dingguo Changsheng Biotechnology Co., Ltd., Beijing, China). To measure the expression levels of ABCA1, ABCG1, SREBP-2, Pcsk9 and LDL-R proteins, $80 \mu \mathrm{g}$ of protein samples were separated on a $10 \%$ sodium dodecyl sulfate-polyacrylamide electrophoresis gel (Beijing Solarbio Science and Technology Co., Ltd., Beijing, China) and transferred onto polyvinylidene fluoride membranes (EMD Millipore, Billerica, MA, USA). The membranes were blocked with 5\% skin, high protein, high calcium milk powder (Inner Mongolia Yili Industrial Group Co., Ltd., Hohhot, China). The membranes were then incubated over- 
$\mathbf{A}$

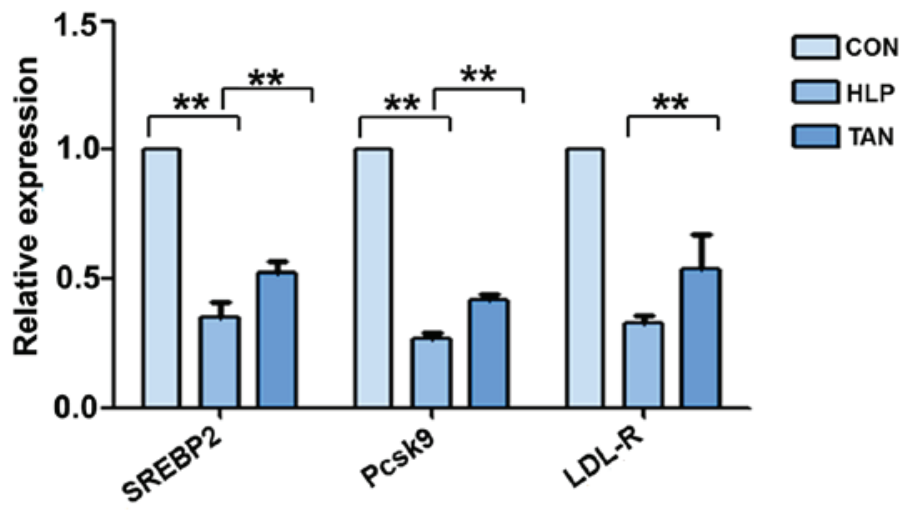

B
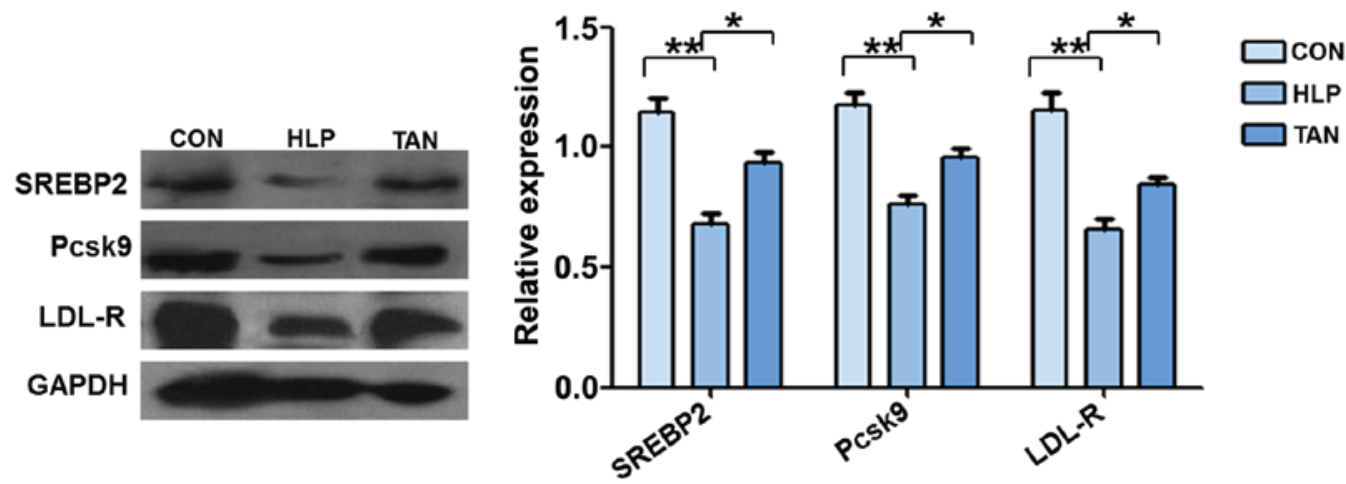

Figure 3. Tanshinone IIA regulation of SREBP-2, Pcsk9 and LDL-R mRNA and protein expression levels in the liver. (A) Reverse transcription-polymerase chain reaction measurement of SREBP-2, Pcsk9 and LDL-R mRNA expression levels in rat liver tissue. (B) Western blotting analysis of SREBP-2, Pcsk9 and LDL-R protein expression levels in the liver. Data are presented as the mean \pm standard deviation. ${ }^{*} \mathrm{P}<0.05$, $^{* *} \mathrm{P}<0.01$. SREBP-2, sterol regulatory element-binding protein 2; Pcsk9, proprotein convertase subtilisin/kexin type 9; LDL-R, low-density lipoprotein receptor; CON, control; HLP, high lipid diet-fed rats; TAN, Tanshinone IIA treatment of high lipid diet-fed rats; GAPDH, .glyceraldehyde 3-phosphate dehydrogenase.

night at $37^{\circ} \mathrm{C}$ with rabbit anti-rat SREBP-2, LDL-R, PCSK9, ABCA1 (cat no. bs-1627R), ABCG1 (cat no. bs-1231R) and GAPDH (No. bs-2188R) primary antibodies (all 1:300; BIOSS), and then with the HRP-conjugated goat anti-rabbit secondary antibody $(1: 200)$ for $1 \mathrm{~h}$ at $37^{\circ} \mathrm{C}$. Protein bands were visualized using an enhanced chemiluminescence kit (Thermo Fisher Scientific, Inc.) and exposed to X-ray films (Kodak, Rochester, NY, USA). Protein expression levels were normalized to GAPDH levels.

Statistical analysis. Data were expressed as the mean \pm standard deviation. Comparisons between the group values were performed by one-way analysis of variance. Tukey's test was used for multiple comparisons between the groups. $\mathrm{P}<0.05$ was considered to indicate a statistically significant difference.

\section{Results}

Tanshinone IIA alterations in lipid levels in sera and lipid deposition in the liver. In the present study, an in vivo experiment was performed to assess the effects of Tanshinone IIA on alterations in the lipid levels in sera and lipid deposition in the liver. The results indicated that compared with the control rats, the HLP rats had significantly higher serum levels of TG, TC and LDL-C, however, significantly lower serum levels of HDL-C $(\mathrm{P}<0.05$ or $\mathrm{P}<0.01)$. Compared with the HLP group, the TAN group did not exhibit altered lipid profiles (Fig. 1A).
Furthermore, H\&E stained liver sections indicated that the hepatic cord is arranged normally in the control rats and that hepatocytes display normal morphology, with a large round nucleus in the centre and little accumulation of lipid droplets in the cytoplasm. There was no infiltration of inflammatory cells in the lobules. However, in the HLP rats, severe hepatic steatosis was observed, and hepatocytes were swollen and round, and the cytoplasm was loose and contained large lipid droplets (Fig. 1B). In a number of hepatocytes, the nucleus was located in the periphery, and lipid vacuoles of varying size and inflammatory cells were observed. In the TAN rats, the level of hepatic steatosis, the number of lipid vacuoles and hepatocyte size were all reduced (Fig. 1B). The oil red $\mathrm{O}$ staining indicated that the hepatocytes in the HLP rats had significantly greater numbers of lipid droplets compared with the control rats, whereas the number of lipid droplets in hepatocytes was significantly reduced in the TAN rats (Fig. 1C).

Tanshinone IIA regulation of $\mathrm{miR}-33 a, A B C A 1$ and $A B C G 1$ expression in the liver. The expression levels of miR-33a in the livers of HLP rats was significantly upregulated compared with the control rats $(\mathrm{P}<0.01)$, whereas the expression levels of miR-33a were significantly reduced in the TAN rats $(\mathrm{P}<0.01$; Fig. 2A), indicating that Tanshinone IIA was able to control miR-33a expression in the rat liver.

Furthermore, expression of ABCA1 mRNA and protein in the liver of HLP rats was significantly downregulated 
A
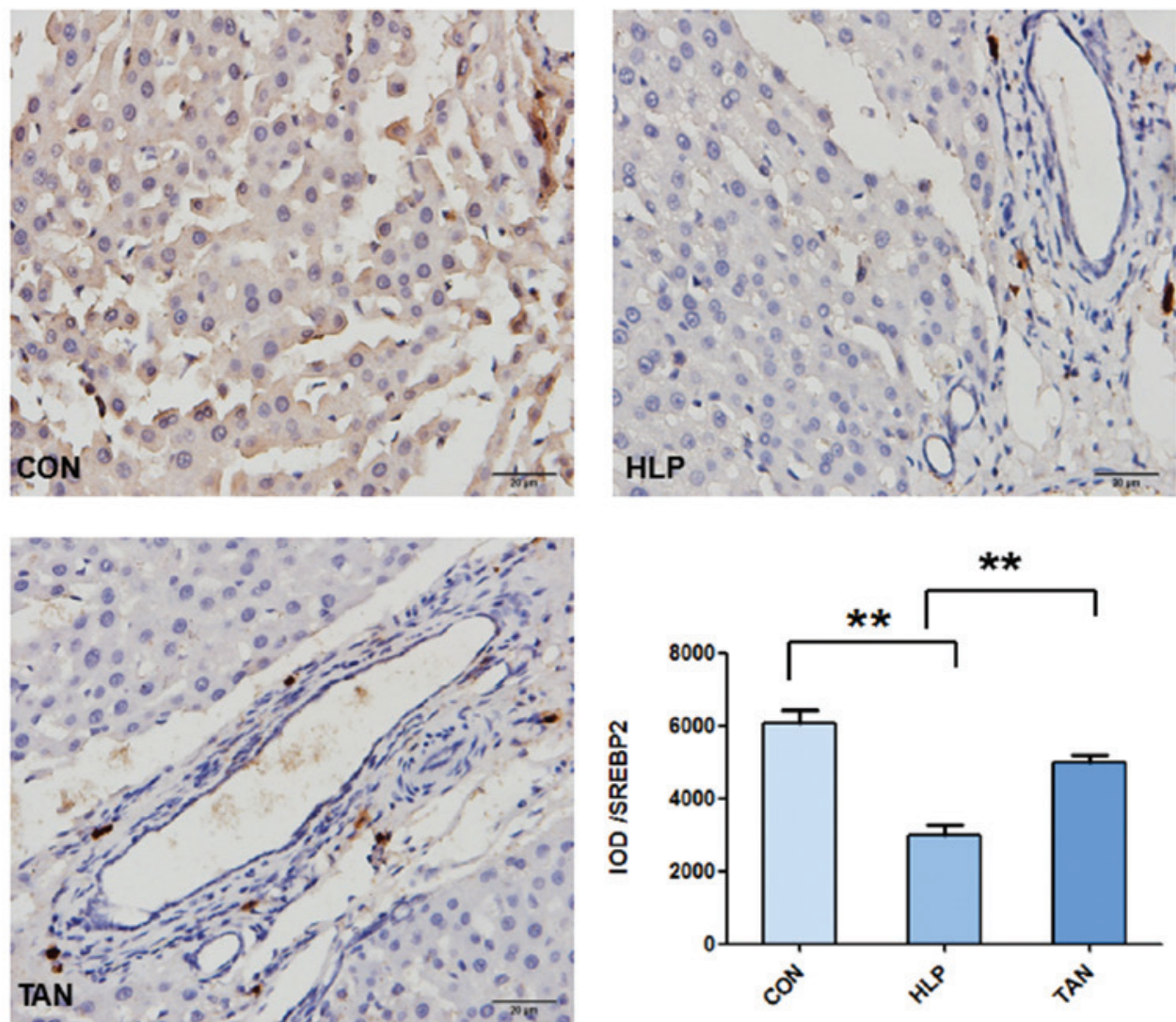

B
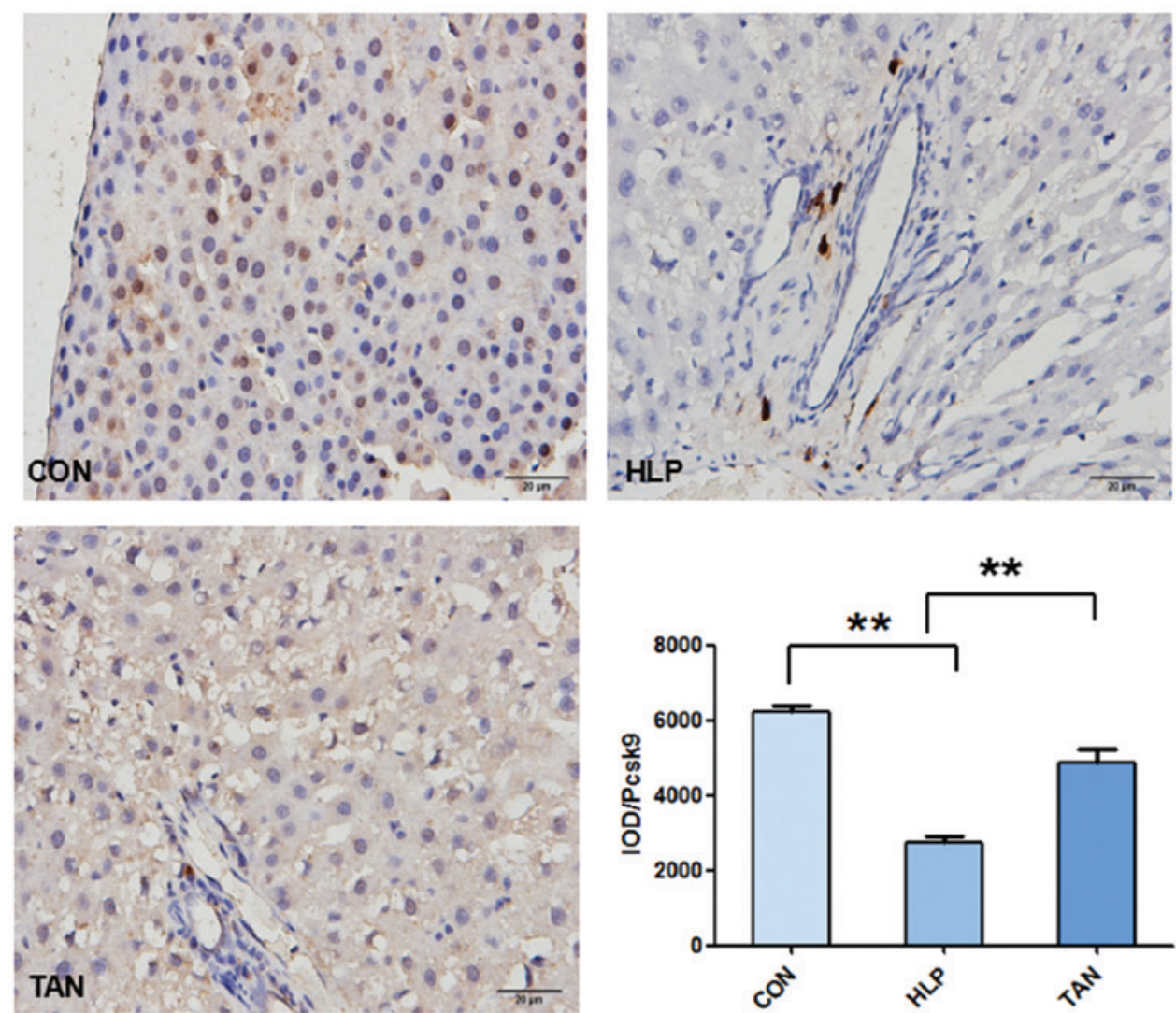

Figure 4. Immunohistochemical detection of SREBP-2, Pcsk9 and LDL-R expression in the liver tissue. Tissue from the CON, HLP and TAN groups was collected and stained with antibodies against SREBP-2, Pcsk9 and LDL-R. (A) SREBP-2 protein expression in liver tissue. (B) Pcsk9 protein expression in liver tissue. Data are presented as the mean \pm standard deviation. ${ }^{* *} \mathrm{P}<0.01$. SREBP-2, sterol regulatory element-binding protein 2; Pcsk9, proprotein convertase subtilisin/kexin type 9; LDL-R, low-density lipoprotein receptor; CON, control; HLP, high lipid diet-fed rats; TAN, Tanshinone IIA treatment of high lipid diet-fed rats; IOD, integrated optical density. 
$\mathbf{C}$
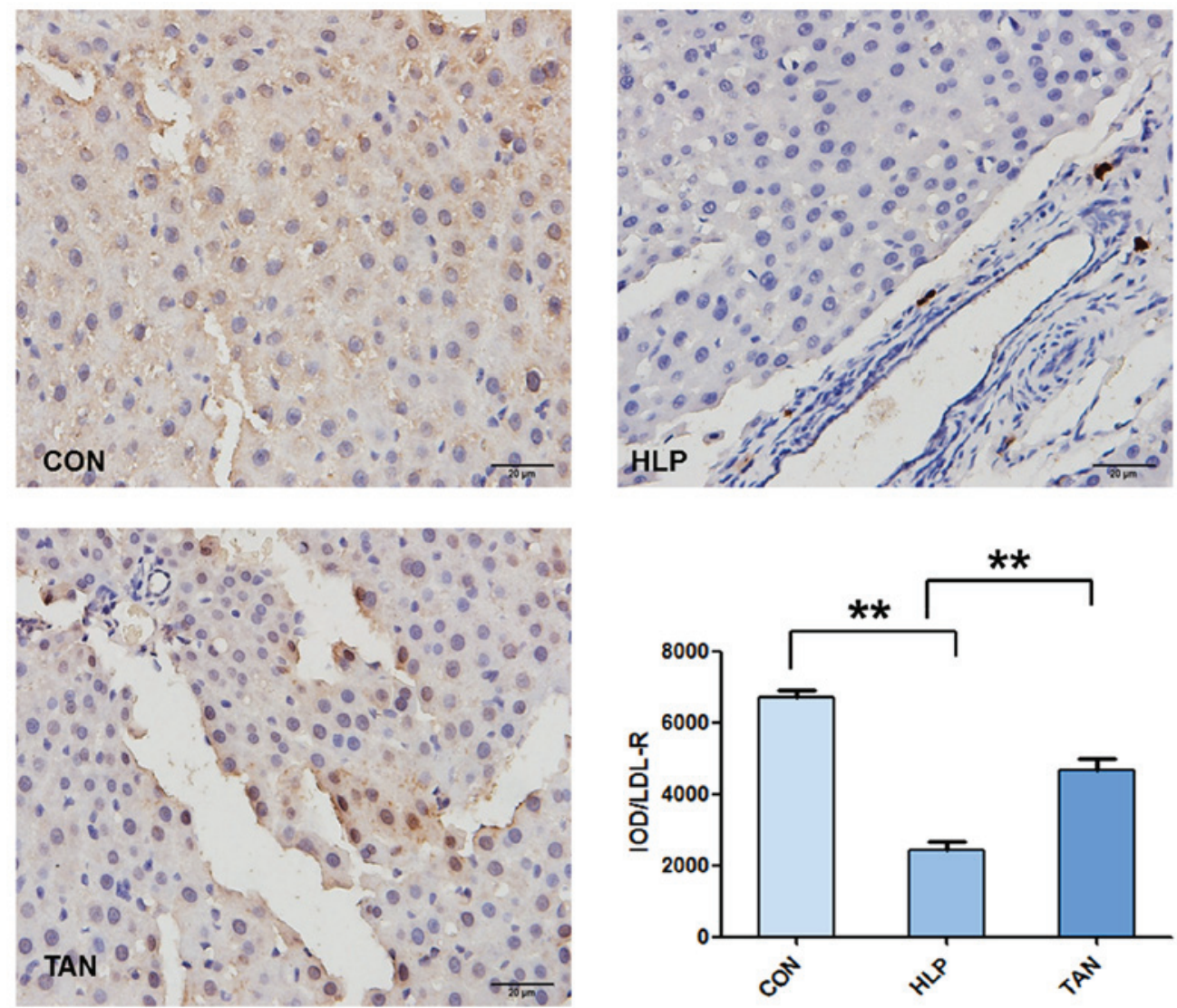

Figure 4 continued. (C) LDL-R protein expression in liver tissue. Data are presented as the mean \pm standard deviation. ** $\mathrm{P}<0.01$. LDL-R, low-density lipoprotein receptor; CON, control; HLP, high lipid diet-fed rats; TAN, Tanshinone IIA treatment of high lipid diet-fed rats; IOD, integrated optical density.

compared with the control rats $(\mathrm{P}<0.01)$, whereas the expression levels of ABCA1 protein were significantly higher in the TAN rats compared with the HLP rats $(\mathrm{P}<0.05)$, however, the levels of ABCA1 mRNA showed no significant alteration (Fig. 2B). In addition, the expression levels of ABCG1 mRNA and protein showed no significant alterations between the TAN and HLP rats (Fig. 2B).

Tanshinone IIA regulation of SREBP-2, Pcsk9 and LDL-R $m R N A$ and protein expression in the liver. The mRNA expression levels of SREBP-2, Pcsk9 and LDL-R were significantly reduced in the liver tissue of HLP rats compared with the control rats $(\mathrm{P}<0.01$; Fig. 3A). However, the mRNA expression levels of SREBP-2 and Pcsk9 were significantly upregulated in the liver tissue of TAN rats, with no alterations observed in LDL-R mRNA expression. The western blotting data supported the RT-qPCR data (Fig. 3B). The immunohistochemical staining additionally indicated that the liver tissues from the HLP rats exhibited weak staining for SREBP-2, Pcsk9 and LDL-R compared with the control rats, whereas the liver tissues from the TAN rats indicated stronger staining for SREBP-2, Pcsk9 and LDL-R ( $\mathrm{P}<0.01$; Fig. 4).

\section{Discussion}

Tanshinone is an active compound in Danshen, a traditional Chinese medicine, which has been used historically for the prevention and treatment of cardiovascular diseases in China.
There are four major lipophilic components of Tanshinone, namely Tanshinone I, Tanshinone IIA, Tanshinone IIB and crypto Tanshinone. Of these, Tanshinone IIA is the most abundant derivative and structural representative of the Tanshinones (7). In the current study, the in vivo effects of Tanshinone IIA were investigated in a rat model of hyperlipidemia, which indicated that Tanshinone IIA was able to reduce lipid deposition in the liver, without altering the blood lipid profile following a three-month treatment. At the gene level, Tanshinone IIA inhibited miR-33a expression in the liver and upregulated ABCA1, ABCG1, SREBP-2 and Pcsk9 expression. These genes may form a miR-33a/SREBP-2/Pcsk9 signaling pathway to regulate lipid metabolism (27-29). Thus, the current study indicates that Tanshinone IIA should be further studied due to their potential use clinically to prevent or treat fatty liver and associated diseases.

Fat-enriched diets have been used for decades to model obesity, hyperlipidemia, dyslipidemia and insulin intolerance in rodents (30). In the current study, rats were fed with a high-fat diet for three months to establish a rat model of hyperlipidemia according to previous studies (31). Following 3 months of a high fat diet, a group of rats were treated with Tanshinone IIA for a further 3 months. The aim of the present study was to demonstrate Tanshinone IIA activity against hyperlipidemia in vivo, with the data indicating that Tanshinone IIA is able to reduce lipid deposition in the liver, however, did not affect the serum lipid profile. Clinically, lipid deposition in the liver can result in fatty liver, abnormal liver function or cirrhosis, 
which may be due to non-alcoholic fatty liver disease or excessive alcohol consumption. The prevalence of non-alcoholic fatty liver disease ranges from $9-36.9 \%$ of the population in different parts of the world (32-34). From use in Chinese clinics, Danshen has been observed to display no notable side effects; thus, Tanshinone IIA may have potential in aiding the control of fatty livers.

Cholesterol is an essential structural component of the cell membrane in the majority of vertebrates, and its transportation requires lipoproteins in the human body (35). It is known that LDL is the main carrier of cholesterol in the blood, and altered serum LDL levels are considered a major risk factor for cardiovascular disease (36). LDL-R, a transmembrane glycoprotein, is the predominant molecule to maintain serum LDL levels and serves an important role in cholesterol homeostasis $(37,38)$. When cells accumulate excess sterols, LDL-R expression on the cell surface will reduced. By contrast, upon depletion of intracellular cholesterol, cells express a high level of LDL-R (39). Previous studies have indicated that Pcsk9 is highly expressed in the liver and contributes to cholesterol homeostasis by regulating LDL-R levels $(39,40)$. Pcsk9 mutations have been associated with autosomal dominant hypercholesterolemia, which is characterized by high LDL levels (41). In support of this, the current study indicated that Tanshinone IIA is able to promote Psck9 expression in liver tissue. miRNAs are a class of non-coding small RNAs, 18-22 nucleotides long, which serve an important role in the regulation of gene expression at the post-transcriptional level $(42,43)$. Previous studies have demonstrated that miR-33 is able to regulate cholesterol efflux and HDL biogenesis by suppressing expression of the ABC transporters, ABCA1 and ABCG1 $(39,26)$. These transporters promote the efflux of phospholipids and cholesterol to their associated apolipoprotein, apo-A1, to generate nascent, discoidal HDL particles, a critical step for the initiation of reverse cholesterol transport to the liver for excretion (5). A previous study demonstrated that there are three highly conserved miR-33 binding sites in the 3'-untranslated region of ABCA1, and that miR-33 overexpression represses the expression of $\mathrm{ABCA} 1$ protein in a variety of cells (20). SREBPs are transcription factors that bind to the sterol regulatory element DNA sequence, TCACNCCAC, to regulate the cholesterol biosynthetic pathway (44). Hepatic cholesterol deprivation results in SREBP cleavage-activating protein escorting SREBP to the Golgi apparatus for processing and activation, followed by transportation to the nucleus and the upregulation of target gene expressions $(45,46)$. In the current study, miR-33a expression was observed to be upregulated, whilst the expression levels of ABCA1 and ABCG1 were reduced in the liver tissues of HLP rats, which further supports that ABCA1 and ABCG1 are the miR-33a target genes. Thus, further studies should investigate whether the effect of Tanshinone IIA is mediated by suppression of miR-33a expression.

To conclude, the current study indicates that Tanshinone IIA is able to attenuate lipid deposition in the livers of hyperlipidemic rats, and modulate the expression of miR-33a and SREBP2/Pcsk9 signaling pathway proteins. Further studies are required to investigate the underlying molecular mechanisms and to assess the potential clinical use of Tanshinone IIA.

\section{Acknowledgements}

The current study was supported by the National Natural Science Foundation of China (grant no. 81202834), National Program on Key Basic Research Project (973 Program) (grant no. 2013CB531704), Natural Science Foundation of Liaoning Province (grant no. 2015020394), and Institutions of Higher Learning Talents Support Program in Liaoning Province (grant no. LR2015041).

\section{References}

1. Chait A and Brunzell JD: Acquired hyperlipidemia (secondary dyslipoproteinemias). Endocrinol Metab Clin North Am 19: 259-278, 1990

2. Williams AD: Hyperlipidaemia and atherogenesis. Med Hypotheses 33: 213-217, 1990.

3. Xie Y, He YB, Zhang SX, Pan AQ, Zhang J, Guan XH, Wang JX and Guo WS: Treatment of combined hyperlipidemia patients by jiangzhi tongluo soft capsule combined atorvastatin calcium tablet: A clinical study. Chin J Int Trad Western Med 34: 1059-1063, 2014 (In Chinese).

4. Fruebis J, Bird DA, Pattison J and Palinski W: Extent of antioxidant protection of plasma LDL is not a predictor of the antiatherogenic effect of antioxidants. J Lipid Res 38: 2455-2464, 1997.

5. Tall AR, Yvan-Charvet L, Terasaaka N, Pagler T and Wang N: HDL, ABC transporters and cholesterol efflux: Implications for the treatment of atherosclerosis. Cell Metab 7: 365-375, 2008.

6. Tang F, Wu X, Wang T, Wang P, Li R, Zhang H, Gao J, Chen S, Bao L, Huang $\mathrm{H}$ and Liu P: Tanshinone IIA attenuates atherosclerotic calcification in rat model by inhibition of oxidative stress. Vascul Pharmacol 46: 427-438, 2007.

7. Gao S, Liu Z, Li H, Little PJ, Liu P and Xu S: Cardiovascular actions and therapeutic potential of Tanshinone IIA Atherosclerosis 220: 3-10, 2012.

8. Xu W, Yang J and Wu LM: Cardioprotective effects of anshinone IIA on myocardial ischemia injury in rats. Pharmazie 64: 332-336, 2009.

9. Gong Z, Huang C, Sheng X, Zhang Y, Li Q, Wang MW, Peng L and Zang YQ: The role of Tanshinone IIA in the treatment of obesity through peroxisome proliferator-activated receptor gamma antag-onism. Endocrinology 150: 104-113, 2009.

10. Tang FT, Cao Y, Wang TQ, Wang LJ, Guo J, Zhou XS, Xu SW, Liu WH, Liu PQ and Huang HQ: Tanshinone IIA attenuates atherosclerosis in ApoE $(-/-)$ mice through down-regulation of scavenger receptor expression. Eur J Pharmacol 650: 275-884, 2011.

11. Jia LQ, Feng JY, Yang GL, Chen WN and Chen Y: Effect of Tanshinone IIA on TLR4 and TNF- $\alpha$ of endothelial cells induced by LPS. Chin J Cell Mol Immunol 27: 733-735, 2011 (In Chinese).

12. Jia LQ, Yang GL, Ren L, Chen WN, Feng JY, Gao Y, Zhang L, $\mathrm{Li} \mathrm{XT}$ and Lei P: Tanshinone IIA reduces apoptosis induced by hydrogen peroxide in the human endothelium-derived EA.hy 926 cells. J Ethnopharmacol 143: 100-108, 2012.

13. Horie T, Ono K, Horiguchi M, Nishi H, Nakamura T, Nagao K, Kinoshita M, Kuwabara Y, Marusawa H, Iwanaga Y, et al: MicroRNA-33 encoded by an intron of sterol regulatory element-binding protein 2 (SREBP-21) regulates HDL in vivo. Proc Natl Acad Sci USA 107: 17321-17326, 2010.

14. Dávalos A, Goedeke L, Smibert P, Ramirez CM, Warrier NP, Andreo U, Cirera-Salinas D, Rayner K, Suresh U, Pastor-Pareja JC, et al: miR-33a/b contribute to the regulation of fatty acid metabolism and insulin signaling. Proc Natl Acad Sci USA 108: 9232-9237, 2011.

15. Wu CY, Tang ZH, Liu LS and Jiang ZS: Selecting pharmacological targets of Pcsk9. Chin J Biochem Mol Biol 25: 991-996, 2009 (In Chinese).

16. Costet P, Cariou B, Lambert G, Lalanne F, Lardeux B, Jarnoux AL, Grefhorst A, Staels B and Krempf M: Hepatic Pcsk9 expression is regulated by nutritional status via insulin and sterol regulatory element-binding protein 1c. J Biol Chem 281: 6211-6218, 2006.

17. Attie AD: The mystery of Pcsk9. Arterioscler Thromb Vasc Biol 24: 1337-1339, 2004. 
18. Steinberg D and Witztum JL: Inhibition of Pcsk9: A powerful weapon for achieving ideal LDL cholesterol levels. Proc Natl Acad Sci USA 106: 9546-9547, 2009.

19. Dong B, Wu MH, Li H, Kraemer FB, Adeli K, Seidah NG, Park SW and Liu J: Strong iduction of Pcsk9 gene expression through HNF1alpha and SREBP-2: Mechanism for the resistance to LDL-cholesterol lowering effect of statins in dyslipidemic hamsters. J Lipid Res 51: 1486-1495, 2010.

20. Najafi-Shoushtari SH, Kristo F, Li Y, Shioda T, Cohen DE, Gerszten RE and Näär AM: MicroRNA-33 and the SREBP host genes cooperate to control cholesterol homeostasi. Science 328: 1566-1569, 2010.

21. Attie AD: ABCA1: At the nexus of cholesterol, HDL and atherosclerosis. Trends Biochem Sci 32: 172-179, 2007.

22. Fernández-Hernando C, Ramírez CM, Goedeke L and Suárez Y: MicroRNAs in metabolic disease. Arterioscler Thromb Vasc Bio 33: 178-185, 2013.

23. Gerin I, Clerbaux LA, Haumont O, Lanthier N, Das AK, Burant CF, Leclercq IA, MacDougald OA and Bommer GT: Expression of miR-33 froman SREBP-2 intron inhibits cholesterol export and fatty acid oxidation. J Biol Chem 285: 33652-33661, 2010.

24. Fernández-Hernando C, Suárez Y, Rayner KJ and Moore KJ. MicroRNAs in lipid metabolism. Curr Opin Lipidol 22: 86-92, 2011.

25. Lewis GF and Rader DJ: New insights into the regulation of HDL metabolism and reverse cholesterol transport. Circ Res 96 $1221-1232,2005$

26. Rayner KJ, Sheedy FJ, Esau CC, Hussain FN, Temel RE, Parathath S, van Gils JM, Rayner AJ, Chang AN, Suarez Y, et al: Antagonism of miR-33 in mice promotes reverse cholesterol transport and regression of atherosclerosis. J Clin Invest 121: 2921-2931, 2011

27. Rayner KJ, Esau CC, Hussain FN, McDaniel AL, Marshall SM van Gils JM, Ray TD, Sheedy FJ, Goedeke L, Liu X, et al: Inhibition of $\mathrm{miR}-33 \mathrm{a} / \mathrm{b}$ in non-human primates raises plasma HDL and lowera VLDL triglycerides. Nature 478: 404-447, 2011.

28. Liu W, Zhai C, Zhang X, Zhang H, Jiang M, Zhou S, Liu Y, Zhao $\mathrm{N}$ and Zhao J: Research of the whole grain-soybean compound package to regulate the cholesterol metabolism by SREBP-2, LDLR and visfatin. J Hyg Res 42: 196-202, 2013 (In Chinese).

29. $\mathrm{Xu} \mathrm{W}$, Liu L and Homby D: c-IAP1 binds and processes Pcsk9 protein: Linking the c-IAP1 in a TNF- $\alpha$ pathway to Pcsk9-mediated LDLR degradation pathway. Molecules 17: 12086-12101, 2012.

30. Mani DN, Bawankule D and Saroj BK: Hyperlipidemic model: Studying lipid profile in small experimental animal. Int J Pharmacy Pharm Sci 4: 337-340, 2012.

31. Munshi RP, Joshi SG and Rane BN: Development of an experimental diet model in rats to study hyperlipidemia and insulin resistance, markers for coronary heart disease. Indian J Pharmacol 46: 270-246, 2014.
32. Omagari K, Kadokawa Y, Masuda J, Egawa I, Sawa T, Hazama H, Ohba K, Isomoto H, Mizuta Y, Hayashida K, et al: Fatty liver in non-alcoholic non overweight Japanese adults: Incidence and clinical characteristics. J Gastroenterol Hepatol 17: 1098-1105, 2002.

33. Hilden M, Christoffersen P, Juhl E and Dalgaard JB: Liver histology in a 'normal' population-examinations of 503 consecutive fatal traffic casualties. Scand J Gastroenterol 12: 593-597, 1997.

34. Shen L, Fan JG, Shao Y, Zeng MD, Wang JR, Luo GH, Li JQ and Chen SY: Prevalence of nonalcoholic fatty liver among administrative officers in Shanghai: An epidemiological survey. World J Gastroenterol 9: 1106-1110, 2003.

35. Ikonen E: Cellular cholesterol trafficking and compartmentalization. Nat Rev Mol Cell Biol 9: 125-138, 2008.

36. Sone H, Tanaka S, Tanaka S, Iimuro S, Oida K, Yamasaki Y, Oikawa S, Ishibashi S, Katayama S, Ohashi Y, et al: Serum level of triglycerides is a potent risk factor comparable to LDL cholesterol for coronary heart disease in Japanese patients with type 2 diabetes: Subanalysis of the Japan Diabetes Complications Study (JDCS). J Clin Endocrinol Metab 96: 3448-3456, 2011.

37. Brown MS and Goldstein JL: A receptor-mediated pathway for cholesterol homeostasis. Science 232: 34-47, 1986.

38. Sharpe LJ and Brown AJ: Rapamycin down-regulates LDL-receptor expression independently of SREBP-2. Biochem Biophys Res Commun 373: 670-674, 2008.

39. Rayner KJ, Suárez Y, Dávalos A, Parathath S, Fitzgerald ML, Tamehiro N, Fisher EA, Moore KJ and Fernández-Hernando C: MiR-33 contributes to the regulation of cholesterol homeostasis. Science 328: 1570-1573, 2010.

40. Maxwell KN and Breslow JL: Adenoviral-mediated expression of Pcsk9 in mice results in a low-density lipoprotein receptor knockout phenotype. Proc Natl Acad Sci USA 101: 7100-7105, 2004.

41. Abifadel M, Varret M, Rabès JP, Allard D, Ouguerram K, Devillers M, Cruaud C, Benjannet S, Wickham L, Erlich D, et al: Mutations in Pcsk9 cause autosomal dominant hypercholesterolemia. Nat Genet 34: 154-156, 2003.

42. Krol J, Loedige I and Filipowicz W: The widespread regulation of microRNA biogenesis, function and decay. Nat Rev Genet 11: 597-610, 2010.

43. Kato M, de Lencastre A, Pincus Z and Slack FJ: Dynamic expression of small non-coding RNAs, including novel microRNAs and piRNAs/21U-RNAs, during Caenorhabditis elegans development. Genome Biol 10: R54, 2009.

44. Roglans N, Peris C, Verd JC, Alegret M, Vázquez M, Sánchez RM and Laguna JC: Increase in hepatic expression of SREBP-2 by gemfibrozil administration to rats. Biochem Pharmacol 62: 803-809, 2011.

45. Horton JD and Shimomura I: Sterol regulatory element-binding proteins: Activators of cholesterol and fatty acid biosynthesis Curr Opin Lipidol 10: 143-150, 1999.

46. Horton JD: Sterol regulatory element-binding proteins: Transcriptional activators of lipid synthesis. Biochem Soc Trans 30: 1091-1095, 2002. 